\title{
Global Dissipative Solutions of the Camassa-Holm Equation
}

\author{
Alberto Bressan \\ Department of Mathematics, Pennsylvania State University, University Park 16802, U.S.A. \\ e-mail: bressan@math.psu.edu \\ and \\ Adrian Constantin \\ Department of Mathematics, Lund University, 22100 Lund, Sweden \\ Trinity College, Department of Mathematics, Dublin 2, Ireland \\ e-mail: adrian.constantin@math.lu.se
}

\begin{abstract}
This paper is devoted to the continuation of solutions to the Camassa-Holm equation after wave breaking. By introducing a new set of independent and dependent variables, the evolution problem is rewritten as a semilinear hyperbolic system in an $\mathbf{L}^{\infty}$ space, containing a non-local source term which is discontinuous but has bounded directional variation. For a given initial condition, the Cauchy problem has a unique solution obtained as fixed point of a contractive integral transformation. Returning to the original variables, we obtain a semigroup of global dissipative solutions, defined for every initial data $\bar{u} \in H^{1}(\mathbb{R})$, and continuously depending on the initial data. The new variables resolve all singularities due to possible wave breaking and ensure that energy loss occurs only through wave breaking.
\end{abstract}

\section{0 - Introduction}

The Camassa-Holm equation

$$
u_{t}-u_{t x x}+3 u u_{x}=2 u_{x} u_{x x}+u u_{x x x} \quad t>0, x \in \mathbb{R},
$$

models the propagation of water waves in the shallow water regime, when the wavelength is considerably larger than the average water depth. Here $u(t, x)$ represents the water's free surface over a flat bed $[\mathrm{CH}]$. The equation (0.1) was first considered by Fokas and Fuchssteiner [FF] as an abstract bi-Hamiltonian P.D.E. with infinitely many conservation laws. For a detailed discussion of the conservation laws we refer to $[\mathrm{I}]$ and $[\mathrm{L}]$. This equation attracted a lot of attention after Camassa and Holm $[\mathrm{CH}]$ derived it as a model for shallow water waves and discovered that it is formally integrable, in the sense that there is an associated Lax pair, and that its solitary waves are solitons, i.e. they retain their shape and speed after the interaction with waves of the same type. An alternative derivation of the equation as a model for shallow water waves was subsequently given by Johnson $[\mathrm{J}]$.

The Camassa-Holm equation has a very rich structure. For a large class of initial data the equation is an integrable infinite dimensional Hamiltonian system: to each solution with initial data in this class, one can associate some scattering data that evolve in time linearly at constant 
speed, and from which the solution can be reconstructed explicitly, see [BSS2, CM1, C2]. In contrast to the Korteweg-de Vries equation, which is a classical integrable model for shallow water waves, the Camassa-Holm equation possesses not only solutions that are global in time but models also wave breaking. When this happens, the solution remains Hölder continuous and uniformly bounded, but develops an unbounded slope in finite time [C1]. By looking at a functional of the initial data, one can predict whether solutions will remain globally smooth [CE1] or produce wave breaking in finite time [CE2]. An aspect of considerable interest is the behavior of the solutions after wave breaking. In [XZ1, XZ2] and more recently in [CHK], global dissipative solutions to the Camassa-Holm equations, in a weak distributional sense, were obtained as weak limits of viscous regularizations.

only

In [BC2] a new approach in the analysis of the Camassa-Holm equation was developed. By introducing a new set of independent and dependent variables, the equation was transformed into a semilinear hyperbolic system. This yields an O.D.E. in a suitable Banach space, whose solutions can be obtained as fixed point of a contractive transformation. Returning to the original variables, this provided a group of conservative solutions, globally defined forward and backward in time. Here "conservative" means that the total energy of the solution equals a constant, for almost every time $t \in \mathbb{R}$. An advantage of this approach, besides being more direct, is that it provides solutions in a stronger sense. Namely, these solutions are Lipschitz continuous when regarded as mappings $u:[0, T] \mapsto L^{2}(\mathbb{R})$, and satisfy the equation at a.e. time $t$. A similar approach, based on the introduction of suitable Lagrangian variables, was pursued in [BZZ] and in [BZ], in connection with first and second order nonlinear wave equations respectively.

The aim of this paper is to prove that, by a suitable modification in the definition of the semilinear hyperbolic system, one can construct a continuous semigroup of dissipative solutions forward in time. We remark that the dissipative case is more delicate, because the corresponding O.D.E. now contains a discontinuous non-local source term. The existence and uniqueness of solutions can still be be established, observing that all discontinuities are crossed transversally. More precisely, we will write hyperbolic system as an O.D.E. in an $\mathbf{L}^{\infty}$ space, having locally bounded variation in the direction of a suitable cone. The abstract result in [BS] thus provides the well-posedness of the Cauchy problem.

Reverting to the original coordinates, we obtain a local, dissipative solution of the CamassaHolm equation. Thanks to a uniform bound on the $H^{1}$ norm, this solutions can be extended forward in time for all $t \geq 0$. Our construction yields a continuous semigroup on the space $H^{1}(\mathbb{R})$. We observe that energy loss for our dissipative solutions can occur only during wave breaking. This last feature is physically relevant. Indeed, it is known that while water waves break a certain amount of heat is produced. This is indicative of a loss of energy during the process.

The uniqueness of dissipative solutions to the Camassa-Holm equation is a delicate issue. What we prove here is that:

- Our constructive procedure (via coordinate transformations) yields a unique semigroup of solutions, defined on the entire space $H^{1}$.

- All of our solutions satisfy the Oleinik type inequality

$$
u_{x}(t, x) \leq C\left(1+t^{-1}\right) \quad t>0,
$$

with a constant $C$ depending only on the norm of the initial data $\|\bar{u}\|_{H^{1}}$.

This leaves open the possibility that other constructive procedures yield different dissipative solutions. We believe that this is not the case, and that the inequality (0.2) uniquely characterizes 
our semigroup dissipative solutions. A uniqueness proof might be achieved by constructing a suitable distance functional related to optimal transportation, as in $[\mathrm{BF}]$ and [BC1]. However, this is outside the scope of the present paper.

\section{1 - The basic equations}

We consider the following form of the Camassa-Holm equation

$$
u_{t}+\left(u^{2} / 2\right)_{x}+P_{x}=0
$$

where the source term $P$ is defined as a convolution:

$$
P \doteq \frac{1}{2} e^{-|x|} *\left(u^{2}+\frac{u_{x}^{2}}{2}\right) .
$$

The initial data is specified as

$$
u(0, x)=\bar{u}(x), \quad x \in \mathbb{R},
$$

with $\bar{u} \in H^{1}(\mathbb{R})$, the space of absolutely continuous functions $u \in \mathbf{L}^{2}(\mathbb{R})$ with derivative $u_{x} \in$ $\mathbf{L}^{2}(\mathbb{R})$. This space is naturally endowed with the norm

$$
\|u\|_{H^{1}} \doteq\left(\int_{\mathbb{R}}\left[u^{2}(x)+u_{x}^{2}(x)\right] d x\right)^{1 / 2} .
$$

For $u \in H^{1}(\mathbb{R})$, Young's inequality implies

$$
P=\left(1-\partial_{x}^{2}\right)^{-1}\left(u^{2}+\frac{1}{2} u_{x}^{2}\right) \in H^{1}(\mathbb{R})
$$

Notice that the assumption $u(t, \cdot) \in H^{1}(\mathbb{R})$ implies that the convolution in (1.2) is well defined.

Definition 1. By a solution of the Cauchy problem (1.1)-(1.2) on a time interval $[0, T]$ we mean a Hölder continuous function $u=u(t, x)$ defined on $[0, T] \times \mathbb{R}$ such that $u(t, \cdot) \in H^{1}(\mathbb{R})$ at every $t \in[0, T]$, and the map $t \mapsto u(t, \cdot)$ is Lipschitz continuous from $[0, T]$ into $\mathbf{L}^{2}(\mathbb{R})$ and satisfies the initial condition (1.2) together with the following equality between functions in $\mathbf{L}^{2}(\mathbb{R})$ :

$$
\frac{d}{d t} u=-u u_{x}-P_{x} \quad \text { for a.e. } \quad t \in[0, T] .
$$

For smooth solutions, applying the operator $\left(1-\partial_{x}^{2}\right)$ to both sides of $(1.3)$, we recover the original form (0.1) of the Camassa-Holm equation. Differentiating (1.1) w.r.t. $x$ one obtains

$$
u_{x t}+u u_{x x}+u_{x}^{2}-\left(u^{2}+\frac{u_{x}^{2}}{2}\right)+P=0 .
$$

Multiplying (1.1) by $u$ and (1.4) by $u_{x}$, for smooth solutions we obtain the two conservation laws with source term

$$
\left(\frac{u^{2}}{2}\right)_{t}+\left(\frac{u^{3}}{3}+u P\right)_{x}=u_{x} P
$$




$$
\left(\frac{u_{x}^{2}}{2}\right)_{t}+\left(\frac{u u_{x}^{2}}{2}-\frac{u^{3}}{3}\right)_{x}=-u_{x} P .
$$

In particular, for smooth solutions the energy satisfies the conservation law

$$
\left(\frac{u^{2}}{2}+\frac{u_{x}^{2}}{2}\right)_{t}+\left(\frac{u u_{x}^{2}}{2}+u P\right)_{x}=0 .
$$

Hence the total energy

$$
E(t) \doteq \int_{\mathbb{R}}\left(u^{2}(t, x)+u_{x}^{2}(t, x)\right) d x
$$

is constant in time. As shown in [CE1, CE2], there exist smooth initial data $\bar{u}$ with compact support such that the corresponding solution of (0.1)-(0.2) develops singularities at a finite time $T>0$. For such solutions the energy (1.7) remains constant on $[0, T[$, while

$$
\lim _{t \uparrow T}\left\{\inf _{x \in \mathbb{R}} u_{x}(t, x)\right\}=-\infty .
$$

In [BC2] we showed that the solution can be continued after the breaking time by requiring that the energy remains constant for a.e. $t \geq 0$. The present paper is concerned with global global solutions, where wave breaking might induce a partial or even total loss of energy.

Definition 2. A solution of the Cauchy problem (1.1)-(1.2) is said to be dissipative if it satisfies the inequality $(0.2)$ for some constant $C$, and moreover its energy $E(t)$ in $(1.7)$ is a non-increasing function of time.

\section{2 - An associated semilinear system}

Given an initial data $\bar{u} \in H^{1}(\mathbb{R})$, we begin by introducing an energy variable $\xi \in \mathbb{R}$. This will play the role of a Lagrangian variable, remaining constant along characteristics. Define the increasing map $\xi \mapsto \bar{y}(\xi)$ implicitly by the relation

$$
\int_{0}^{\bar{y}(\xi)}\left(1+\bar{u}_{x}^{2}\right) d x=\xi .
$$

We now write a semilinear system of equations in terms of the independent variables $(t, \xi)$. This was first used in [BC2] to construct global conservative solutions to the Camassa-Holm equation. An appropriate modification of this system will here yield global dissipative solutions.

Assuming for the time being that the solution $u$ remains Lipschitz continuous, denote by $t \mapsto y(t, \xi)$ the characteristic curve starting at $\bar{y}(\xi)$, so that

$$
\frac{\partial}{\partial t} y(t, \xi)=u(t, y(t, \xi)), \quad y(0, \xi)=\bar{y}(\xi) .
$$

Throughout the following, we use the notation

$$
u(t, \xi) \doteq u(t, y(t, \xi)), \quad P(t, \xi) \doteq P(t, y(t, \xi)),
$$

and define the variables $v=v(t, \xi)$ and $q=q(t, \xi)$ as

$$
v \doteq 2 \arctan u_{x}, \quad q \doteq\left(1+u_{x}^{2}\right) \cdot \frac{\partial y}{\partial \xi},
$$


with $u_{x}=u_{x}(t, y(t, \xi))$. Since $v$ is defined up to multiples of $2 \pi$, all subsequent equations involving $v$ will be invariant under addition of multiples of $2 \pi$. Notice that (2.1) yields

$$
q(0, \xi) \equiv 1
$$

Moreover one has the useful identities

$$
\frac{1}{1+u_{x}^{2}}=\cos ^{2} \frac{v}{2}, \quad \frac{u_{x}}{1+u_{x}^{2}}=\frac{1}{2} \sin v, \quad \frac{u_{x}^{2}}{1+u_{x}^{2}}=\sin ^{2} \frac{v}{2},
$$

and

$$
\frac{\partial y}{\partial \xi}=\frac{q}{1+u_{x}^{2}}=\cos ^{2} \frac{v}{2} \cdot q
$$

From (2.6) it follows

$$
y\left(t, \xi^{\prime}\right)-y(t, \xi)=\int_{\xi}^{\xi^{\prime}} \cos ^{2} \frac{v(t, s)}{2} \cdot q(t, s) d s .
$$

Furthermore, we have

$$
\begin{gathered}
P(t, \xi)=P(t, y(t, \xi))=\frac{1}{2} \int_{-\infty}^{\infty} e^{-|y(t, \xi)-x|}\left(u^{2}(t, x)+\frac{1}{2} u_{x}^{2}(t, x)\right) d x, \\
P_{x}(t, \xi)=P_{x}(t, y(t, \xi))=\frac{1}{2}\left(\int_{y(t, \xi)}^{\infty}-\int_{-\infty}^{y(t, \xi)}\right) e^{-|y(t, \xi)-x|}\left(u^{2}(t, x)+\frac{1}{2} u_{x}^{2}(t, x)\right) d x .
\end{gathered}
$$

In the above formulas, performing the change of variables $x=y\left(t, \xi^{\prime}\right)$, we can write the convolution as an integral over the variable $\xi^{\prime}$. Using the identities (2.5)-(2.7), we obtain

$$
P(\xi)=\frac{1}{2} \int_{-\infty}^{\infty} \exp \left\{-\left|\int_{\xi}^{\xi^{\prime}} \cos ^{2} \frac{v(s)}{2} \cdot q(s) d s\right|\right\} \cdot\left[u^{2}\left(\xi^{\prime}\right) \cos ^{2} \frac{v\left(\xi^{\prime}\right)}{2}+\frac{1}{2} \sin ^{2} \frac{v\left(\xi^{\prime}\right)}{2}\right] q\left(\xi^{\prime}\right) d \xi^{\prime}
$$

and

$$
P_{x}(\xi)=\frac{1}{2}\left(\int_{\xi}^{\infty}-\int_{-\infty}^{\xi}\right) \exp \left\{-\left|\int_{\xi}^{\xi^{\prime}} \cos ^{2} \frac{v(s)}{2} \cdot q(s) d s\right|\right\} \cdot\left[u^{2}\left(\xi^{\prime}\right) \cos ^{2} \frac{v\left(\xi^{\prime}\right)}{2}+\frac{1}{2} \sin ^{2} \frac{v\left(\xi^{\prime}\right)}{2}\right] q\left(\xi^{\prime}\right) d \xi^{\prime} .
$$

By (1.1) and (2.2), the evolution equation for $u$ in the new variables $(t, \xi)$ takes the form

$$
\frac{\partial}{\partial t} u(t, \xi)=u_{t}+u u_{x}=-P_{x}(t, \xi)
$$

with $P_{x}$ given at (2.9). On the other hand, from (2.2) and (1.6) we obtain

$$
\begin{aligned}
\frac{d}{d t} \int_{\xi_{1}}^{\xi_{2}} q(t, \xi) d \xi & =\frac{d}{d t} \int_{y\left(t, \xi_{1}\right)}^{y\left(t, \xi_{2}\right)}\left(1+u_{x}^{2}(t, x)\right) d x \\
& =\int_{y\left(t, \xi_{1}\right)}^{y\left(t, \xi_{2}\right)}\left\{\left(1+u_{x}^{2}\right)_{t}+\left[u\left(1+u_{x}^{2}\right)\right]_{x}\right\} d x=\int_{y\left(t, \xi_{1}\right)}^{y\left(t, \xi_{2}\right)}\left(2 u^{2}+1-2 P\right) u_{x} d x .
\end{aligned}
$$


Differentiation w.r.t. $\xi$ yields

$$
\frac{\partial}{\partial t} q(t, \xi)=\left(2 u^{2}+1-2 P\right) \frac{u_{x}}{1+u_{x}^{2}} \cdot q=\left(u^{2}+\frac{1}{2}-P\right) \sin v \cdot q .
$$

Moreover, using (2.2)-(2.3) and (1.4), we obtain

$$
\frac{\partial}{\partial t} v(t, \xi)=\frac{2}{1+u_{x}^{2}}\left[-\frac{u_{x}^{2}}{2}+u^{2}-P\right]=2\left(u^{2}-P\right) \cos ^{2} \frac{v}{2}-\sin ^{2} \frac{v}{2} .
$$

In (2.11) and in (2.12), the function $P=P(t, \xi)$ is computed by (2.8). To obtain global conservative solutions, one proceeds as follows. Given the initial data $\bar{u} \in H^{1}(\mathbb{R})$, consider the corresponding Cauchy problems (2.10)-(2.12) for the variables $(u, v, q)$ in the form of the semilinear system

$$
\left\{\begin{array}{l}
\frac{\partial u}{\partial t}=-P_{x} \\
\frac{\partial v}{\partial t}=\left(u^{2}-P\right)(1+\cos v)-\sin ^{2} \frac{v}{2} \\
\frac{\partial q}{\partial t}=\left(u^{2}+\frac{1}{2}-P\right) \sin v \cdot q
\end{array}\right.
$$

with

$$
\left\{\begin{array}{l}
u(0, \xi)=\bar{u}(\bar{y}(\xi)) \\
v(0, \xi)=2 \arctan \bar{u}_{x}(\bar{y}(\xi)) \\
q(0, \xi)=1
\end{array}\right.
$$

The system (2.13) can be regarded as an O.D.E. in the Banach space

$$
X \doteq H^{1}(\mathbb{R}) \times\left[\mathbf{L}^{2}(\mathbb{R}) \cap \mathbf{L}^{\infty}(\mathbb{R})\right] \times \mathbf{L}^{\infty}(\mathbb{R}),
$$

endowed with the norm

$$
\|(u, v, q)\|_{X} \doteq\|u\|_{H^{1}}+\|v\|_{\mathbf{L}^{2}}+\|v\|_{\mathbf{L}^{\infty}}+\|q\|_{\mathbf{L}^{\infty}}
$$

Observing that the right hand side is locally Lipschitz, one obtains the local existence and uniqueness of solutions by general O.D.E. theory. The local solution can be extended to a global solution since blowup is prevented by the conservation of the energy $E(t)$, expressed in the $(t, \xi)$-variables as

$$
E(t)=\int_{\mathbb{R}}\left(u^{2}(t, \xi) \cos ^{2} \frac{v(t, \xi)}{2}+\sin ^{2} \frac{v(t, \xi)}{2}\right) q(t, \xi) d \xi=E(0), \quad t \geq 0 .
$$

Going back to the original variables $u(t, x)$, in [BC2] the authors constructed a semigroup of global solutions to the Camassa-Holm equation. The solutions constructed via this approach are conservative, in the sense that their total energy, measured by the $H^{1}$ norm of $u(t, \cdot)$, is equal to a constant for a.e. time $t \in \mathbb{R}$.

In order to obtain global dissipative solutions, a modification of the system (2.13) is needed. In essence, we require the following. Assume that, along a given characteristic $t \mapsto y(t, \xi)$, the wave breaks at a first time $t=\tau(\xi)$. Recalling our rescaled variable $v=2 \arctan u_{x}$, this of course means $u_{x}(t, \xi) \rightarrow-\infty$ and $v=2 \arctan u_{x}$, as $t \uparrow \tau(\xi)$. For all $t \geq \tau(\xi)$ we then set $v(t, \xi) \equiv-\pi$ 
and remove the values of $u(t, \xi), v(t, \xi), q(t, \xi)$ from the computation of $P, P_{x}$. More precisely, in the definition of $P, P_{x}$ we now replace $(2.8)-(2.9)$ by

$$
\begin{gathered}
P(\xi)=\frac{1}{2} \int_{\left\{v\left(\xi^{\prime}\right)>-\pi\right\}} \exp \left\{-\left|\int_{\left\{s \in\left[\xi, \xi^{\prime}\right], v(s)>-\pi\right\}} \cos ^{2} \frac{v(s)}{2} \cdot q(s) d s\right|\right\} \\
\cdot\left[u^{2}\left(\xi^{\prime}\right) \cos ^{2} \frac{v\left(\xi^{\prime}\right)}{2}+\frac{1}{2} \sin ^{2} \frac{v\left(\xi^{\prime}\right)}{2}\right] q\left(\xi^{\prime}\right) d \xi^{\prime}, \\
P_{x}(\xi)=\frac{1}{2}\left(\int_{\left\{\xi^{\prime}>\xi, v\left(\xi^{\prime}\right)>-\pi\right\}}-\int_{\left\{\xi^{\prime}<\xi, v\left(\xi^{\prime}\right)>-\pi\right\}}\right) \exp \left\{-\left|\int_{\left\{s \in\left[\xi, \xi^{\prime}\right], v(s)>-\pi\right\}} \cos ^{2} \frac{v(s)}{2} \cdot q(s) d s\right|\right\} \\
\cdot\left[u^{2}\left(\xi^{\prime}\right) \cos ^{2} \frac{v\left(\xi^{\prime}\right)}{2}+\frac{1}{2} \sin ^{2} \frac{v\left(\xi^{\prime}\right)}{2}\right] q\left(\xi^{\prime}\right) d \xi^{\prime} .
\end{gathered}
$$

The system of O.D.E's (2.13) is now replaced by

$$
\left\{\begin{array}{l}
\frac{\partial u}{\partial t}=-P_{x}, \\
\frac{\partial v}{\partial t}=\left\{\begin{array}{cl}
\left(u^{2}-P\right)(1+\cos v)-\sin ^{2} \frac{v}{2} & \text { if } \quad v>-\pi, \\
0 & \text { if } \quad v \leq-\pi,
\end{array}\right. \\
\frac{\partial q}{\partial t}=\left\{\begin{array}{cll}
\left(u^{2}+\frac{1}{2}-P\right) \sin v \cdot q & \text { if } \quad v>-\pi, \\
0 & \text { if } \quad v \leq-\pi .
\end{array}\right.
\end{array}\right.
$$

Notice that, in the dissipative case, the Camassa-Holm equation is reduced to an O.D.E. in a Banach space, where the right hand side is now discontinuous. The discontinuity occurs precisely when $v=-\pi$. We observe that, by the second equation in (2.18), $v$ approaches the value $-\pi$ transversally, i.e. with strictly negative derivative $v_{t} \approx-1$. It is precisely this transversality condition that guarantees the well-posedness of the system (2.18).

\section{3 - Local solutions of the semilinear system}

The Cauchy problem for (2.18) can be written in more compact form as

$$
\begin{gathered}
\frac{\partial}{\partial t} U(t, \xi)=F(U(t, \xi))+G(\xi, U(t, \cdot)) \quad \xi \in \mathbb{R}, \\
U(0, \xi)=\bar{U}(\xi) .
\end{gathered}
$$

Here $U=(u, v, q) \in \mathbb{R}^{3}$, while

$$
F(U)= \begin{cases}\left(0, u^{2}(1+\cos v)-\sin ^{2} \frac{v}{2},\left(u^{2}+\frac{1}{2}\right) \sin v \cdot q\right) & \text { if } \quad v>-\pi \\ (0,-1,0) & \text { if } \quad v \leq-\pi\end{cases}
$$




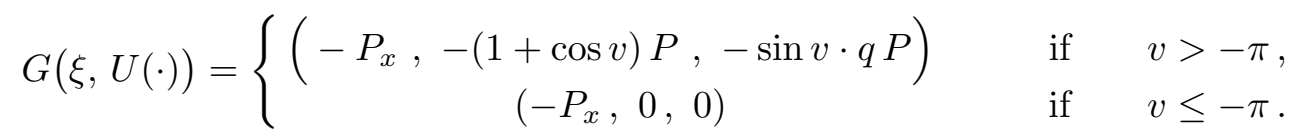

The nonlocal operators $P, P_{x}$ are defined at (2.16)-(2.17).

Notice the slight discrepancy between the systems (3.1)-(3.4) and (2.18). To achieve exactly the same evolution, one should define $F=(0,0,0)$ whenever $v \leq-\pi$. The modification in (3.3) has the advantage of rendering the field $F$ continuous across the value $v=-\pi$. Moreover, as soon as a solution $(u, v, p)$ of $(3.1)-(3.4)$ is obtained, the mapping

$$
(t, \xi) \mapsto(u(t, \xi), \max \{v(t, \xi),-\pi\}, q(t, \xi))
$$

provides a solution of (2.18). We regard (3.1) as an O.D.E. on the space $\mathbf{L}^{\infty}\left(\mathbb{R} ; \mathbb{R}^{3}\right)$. Notice that the vector field $F: \mathbb{R}^{3} \mapsto \mathbb{R}^{3}$ in (3.3) is uniformly bounded and Lipschitz continuous as long as $u$ remains in a bounded set. However, the nonlocal operator $G$ is discontinuous. Indeed, the integral terms $P, P_{x}$ defined at $(2.16),(2.17)$ are discontinuous, because the set

$$
\{\xi ; \tau(\xi)>t\}=\{\xi ; v(t, \xi)>-\pi\}
$$

may suddenly shrink, at a time $\tau^{*}$ such that

$$
\operatorname{meas}\left(\left\{\xi ; v\left(\tau^{*}, \xi\right)=-\pi\right\}\right)>0 .
$$

Aim of this section is to prove:

Theorem 1. Let any $\bar{u} \in H^{1}(\mathbb{R})$ be given. In connection with the corresponding initial data $\bar{U} \doteq\left(\bar{u}, 2 \arctan \bar{u}_{x}, 1\right)$ defined at (2.14), the Cauchy problem (3.1)-(3.2) has a unique local solution, defined on some time interval $[0, T]$.

Proof. The local (and even global) existence and uniqueness of the solution can be obtained by applying the general theorem on directionally continuous O.D.E's in functional spaces recently proved in $[\mathrm{BS}]$. We shall give here a self-contained proof, in several steps, still following [BS] in the main lines.

1. We begin with some a priori estimates on $F$ and $G$ in (3.1). Assume that $U=(u, v, q) \in$ $\mathbf{L}^{\infty}\left(\mathbb{R} ; \mathbb{R}^{3}\right)$ satisfies the inequalities

$$
\begin{gathered}
\|u\|_{\mathbf{L}^{\infty}} \leq C, \quad \frac{1}{C} \leq q(\xi) \leq C \text { for all } \xi, \\
\text { meas }\left(\left\{\xi ; \quad v(\xi)>-\pi, \quad|v(\xi)| \geq \frac{\pi}{2}\right\}\right) \leq C .
\end{gathered}
$$

for some constant $C$. Then there is a constant $\kappa^{*}$ depending only on $C$ such that

$$
\begin{gathered}
\|P\|_{\mathbf{L}^{\infty}}+\left\|P_{x}\right\|_{\mathbf{L}^{\infty}} \leq \kappa^{*}, \quad\|P\|_{\mathbf{L}^{1}}+\left\|P_{x}\right\|_{\mathbf{L}^{1}} \leq \kappa^{*}\left(1+\|u\|_{\mathbf{L}^{1}}+\|v\|_{\mathbf{L}^{1}}\right), \\
\|F(U)\|_{\mathbf{L}^{\infty}} \leq \kappa^{*}, \quad\|G(U)\|_{\mathbf{L}^{\infty}} \leq \kappa^{*} .
\end{gathered}
$$

Moreover, there exists a Lipschitz constant $\kappa$ such that, if $\widetilde{U}=(\tilde{u}, \tilde{v}, \tilde{q})$ satisfies the same bounds (3.7)-(3.8), then

$$
\|F(U)-F(\widetilde{U})\|_{\mathbf{L}^{\infty}} \leq \kappa\|U-\widetilde{U}\|_{\mathbf{L}^{\infty}},
$$




$$
\begin{aligned}
& \|G(U)-G(\widetilde{U})\|_{\mathbf{L}^{\infty}} \leq \kappa \cdot\left[\|U-\widetilde{U}\|_{\mathbf{L}^{\infty}}+\operatorname{meas}(\{\xi ; \quad v(\xi)>-\pi, \quad \tilde{v}(\xi) \leq-\pi\})\right. \\
& +\operatorname{meas}(\{\xi ; \tilde{v}(\xi)>-\pi, \quad v(\xi) \leq-\pi\})] .
\end{aligned}
$$

2. Let $\bar{u} \in H^{1}$ be given. Since $\bar{u}, \bar{u}_{x} \in \mathbf{L}^{2}$, for every $\varepsilon>0$ the set

$$
\left\{x \in \mathbb{R} ;\left|\bar{u}_{x}(x)\right| \geq \varepsilon\right\}
$$

has finite measure. We can thus find a constant $C>0$ such that

$$
\|\bar{u}\|_{\mathbf{L}^{\infty}} \leq \frac{C}{2}, \quad \operatorname{meas}\left(\left\{\xi ;|\bar{v}(\xi)| \geq \frac{\pi}{4}\right\}\right) \leq \frac{C}{2} .
$$

3. Let the inequalities (3.7)-(3.8) hold. By Step 1, we can choose $\delta>0$ small enough so that $v(\xi) \in]-\pi, \delta-\pi]$ implies

$$
\frac{\partial}{\partial t} v(t, \xi)=\left(u^{2}-P\right)(1+\cos v)-\sin ^{2} \frac{v}{2} \leq-\frac{1}{2} .
$$

Consider the sets

$$
\left.\left.\Omega^{\delta} \doteq\{\xi \in \mathbb{R} ; \quad \bar{v}(\xi) \in]-\pi, \delta-\pi\right]\right\}, \quad \Omega^{\prime} \doteq \mathbb{R} \backslash \Omega^{\delta} .
$$

By possibly reducing the size of $\delta>0$, we can assume that

$$
\text { meas }\left(\Omega^{\delta}\right) \leq \frac{1}{8 \kappa}
$$

4. The solution $t \mapsto U(t)$ will be obtained as the unique fixed point of a contractive transformation $\mathcal{P}: \mathcal{D} \mapsto \mathcal{D}$, on a suitable domain $\mathcal{D} \subset \mathcal{C}\left([0, T], \mathbf{L}^{\infty}\right)$. More precisely, for a given $T>0$, we define the domain $\mathcal{D}$ as the set of all continuous mappings $t \mapsto U(t)=(u(t), v(t), q(t))$ from $[0, T]$ into $\mathbf{L}^{\infty}\left(\mathbb{R}, \mathbb{R}^{3}\right)$ with the following properties.

$$
\begin{gathered}
U(0)=\bar{U} \\
\|U(t)-U(s)\|_{\mathbf{L}^{\infty}} \leq 2 \kappa^{*}|t-s|, \\
v(t, \xi)-v(s, \xi) \leq-\frac{t-s}{2} \quad \xi \in \Omega^{\delta}, \quad 0 \leq s<t \leq T .
\end{gathered}
$$

The Picard operator $\mathcal{P}$ is defined as

$$
(\mathcal{P}(U))(t, \xi) \doteq \bar{U}+\int_{0}^{t}[F(U(\tau, \xi))+G(\xi, U(\tau, \cdot))] d \tau .
$$

5. Choosing $T>0$ sufficiently small, it is clear that $\mathcal{P}$ maps the domain $\mathcal{D}$ into itself. We now show that $\mathcal{P}$ is a strict contraction. Indeed, assume $U, \widetilde{U} \in \mathcal{D}$ and define

$$
\eta \doteq \max _{t \in[0, T]}\|U(t)-\widetilde{U}(t)\|_{\mathbf{L}^{\infty}} .
$$


Define the crossing time

$$
\tau(\xi) \doteq \sup \{t \in[0, T] ; \quad v(t, \xi)>-\pi\} .
$$

Define $\tilde{\tau}(\xi)$ in the same way, replacing $v$ by $\tilde{v}$. Observe that, for each $\xi \in \Omega^{\delta}$, the property (3.14) implies

$$
|\tau(\xi)-\tilde{\tau}(\xi)| \leq 2 \eta
$$

For $t \in[0, T]$ we then have

$$
\begin{aligned}
& \|\mathcal{P} U(t)-\mathcal{P} \widetilde{U}(t)\|_{\mathbf{L}^{\infty}} \leq \int_{0}^{t}\|F(U(\tau))-F(\widetilde{U}(\tau))\|_{\mathbf{L}^{\infty}} d \tau+\int_{0}^{t}\|G(U(\tau))-G(\widetilde{U}(\tau))\|_{\mathbf{L}^{\infty}} d \tau \\
& \leq 2 \kappa \cdot \int_{0}^{t}\|U(\tau)-\widetilde{U}(\tau)\|_{\mathbf{L}^{\infty}} d \tau+\kappa \cdot \int_{0}^{t} \operatorname{meas}(\{\xi ; \quad v(\tau, \xi)>-\pi, \quad \tilde{v}(\tau, \xi) \leq-\pi\}) d \tau \\
& \quad+\kappa \cdot \int_{0}^{t} \operatorname{meas}(\{\xi ; \quad \tilde{v}(\tau, \xi)>-\pi, \quad v(\tau, \xi) \leq-\pi\}) d \tau \\
& \leq 2 \kappa T \eta+\kappa \cdot \int_{\Omega^{\delta}} 2|\tau(\xi)-\tilde{\tau}(\xi)| d \xi \\
& \leq 2 \kappa T \eta+2 \kappa \cdot \operatorname{meas}\left(\Omega^{\delta}\right) \cdot \eta \leq \frac{\eta}{2},
\end{aligned}
$$

provided that $T$ is small enough. This proves that $\mathcal{P}$ is a strict contraction, hence it has a unique fixed point, which yields the desired local solution of the Cauchy problem (3.1)-(3.2).

\section{4 - Global solutions}

In this section we show that the local solutions of the semilinear system (2.18) can be globally extended for all times $t \geq 0$. The basic ingredient is a global bound on the total energy:

$$
E(t)=\int_{\{v(t, \xi)>-\pi\}}\left(u^{2}(t, \xi) \cos ^{2} \frac{v(t, \xi)}{2}+\sin ^{2} \frac{v(t, \xi)}{2}\right) q(t, \xi) d \xi
$$

We begin by showing that, as long as the local solution of (2.18) is defined,

$$
u_{\xi}=\frac{q}{2} \sin v
$$

Indeed, the first equation in (2.18) and the definition of $P_{x}$ at (2.17) imply

$$
u_{\xi t}=-\frac{\partial}{\partial \xi} P_{x}=\left\{\begin{array}{lll}
q\left(u^{2} \cos ^{2} \frac{v}{2}+\frac{1}{2} \sin ^{2} \frac{v}{2}-P \cos ^{2} \frac{v}{2}\right) & \text { if } & v(t, \xi)>-\pi \\
0 & \text { if } & v(t, \xi)>-\pi .
\end{array}\right.
$$

On the other hand, from the last two equations in (2.18), when $v>-\pi$ we obtain

$$
\begin{aligned}
\left(\frac{q}{2} \sin v\right)_{t}= & \frac{q_{t}}{2} \sin v+\frac{q}{2} v_{t} \cos v \\
= & \frac{q}{2}\left(\left(u^{2}+\frac{1}{2}-P\right) \sin ^{2} v+\left(u^{2}-P\right) \cos v\right. \\
& \left.\quad+\left(u^{2}-P\right) \cos ^{2} v-\cos v \cdot \sin ^{2} \frac{v}{2}\right) \\
= & q\left(u^{2} \cos ^{2} \frac{v}{2}+\frac{1}{2} \sin ^{2} \frac{v}{2}-P \cos ^{2} \frac{v}{2}\right)
\end{aligned}
$$


while, if $v=-\pi$, the same equations in (2.18) yield

$$
\left(\frac{q}{2} \sin v\right)_{t}=0
$$

Next, at the initial time $t=0$, by (2.14) and (2.5)-(2.6) we have

$$
\frac{\partial u}{\partial \xi}=\bar{u}_{x} \cdot \frac{\partial \bar{y}}{\partial \xi}=\bar{u}_{x} \cdot \frac{1}{1+\bar{u}_{x}^{2}}=\frac{\sin v}{2}=\frac{q}{2} \sin v
$$

because $\bar{q} \equiv 1$. By (4.6), the identity (4.2) holds at time $t=0$. By (4.3)-(4.5), for every $\xi$ we have

$$
\frac{\partial}{\partial t}\left(u_{\xi}-\frac{q \sin v}{2}\right)=0
$$

Hence the identity (4.2) holds for all times $t \geq 0$, as long as the solution is defined.

Next, we claim that the "extended energy"

$$
\widetilde{E}(t) \doteq \int_{\mathbb{R}}\left(u^{2} \cos ^{2} \frac{v}{2}+\sin ^{2} \frac{v}{2}\right) q d \xi
$$

remains constant in time. We remark that the integral in (4.7) in general is strictly larger than the energy $E(t)$ in (4.1), because here the integration ranges over the entire real line. From (2.18) we deduce that

$$
\begin{aligned}
& \frac{d}{d t} \int_{\mathbb{R}}\left(u^{2} \cos ^{2} \frac{v}{2}+\sin ^{2} \frac{v}{2}\right) q d \xi \\
& =\int_{\{v(\xi)>-\pi\}}\left\{2\left(u^{2} \cos ^{2} \frac{v}{2}+\sin ^{2} \frac{v}{2}\right) \cdot\left(u^{2}+\frac{1}{2}-P\right) \sin \frac{v}{2} \cdot \cos \frac{v}{2}-2 u P_{x} \cos ^{2} \frac{v}{2}\right. \\
& \left.\quad \quad+\sin \frac{v}{2} \cdot \cos \frac{v}{2} \cdot\left(1-u^{2}\right)\left[2\left(u^{2}-P\right) \cos ^{2} \frac{v}{2}-\sin ^{2} \frac{v}{2}\right]\right\} q d \xi \\
& =\int_{\mathbb{R}}\left\{-2 P \sin \frac{v}{2} \cdot \cos \frac{v}{2}-2 u P_{x} \cos ^{2} \frac{v}{2}+3 u^{2} \sin \frac{v}{2} \cdot \cos \frac{v}{2}\right\} q d \xi
\end{aligned}
$$

Notice that on the right hand side of (4.8) we are again integrating over the entire real line. Of course, this does not make a difference because $\cos \frac{v}{2}=0$ whenever $v=-\pi$.

On the other hand, from (2.16)-(2.17) we infer

$$
P_{\xi}=q P_{x} \cos ^{2} \frac{v}{2} .
$$

Notice that the case $v=-\pi$ a separate computation yields $P_{\xi}=0$. This is still consistent with (4.9), because $\cos ^{2}(-\pi / 2)=0$.

Together, (4.2) and (4.9) yield

$$
(u P)_{\xi}=u_{\xi} P+u P_{\xi}=\left\{P \sin \frac{v}{2} \cdot \cos \frac{v}{2}+u P_{x} \cos ^{2} \frac{v}{2}\right\} q .
$$

In addition, we observe that

$$
3 q u^{2} \sin \frac{v}{2} \cdot \cos \frac{v}{2}=3 u^{2} u_{\xi}=\left(u^{3}\right)_{\xi} .
$$


Using the above identities, we obtain

$$
\frac{d}{d t} \int_{\mathbb{R}}\left(u^{2} \cos ^{2} \frac{v}{2}+\sin ^{2} \frac{v}{2}\right) q d \xi=\int_{\mathbb{R}} \frac{\partial}{\partial_{\xi}}\left\{u^{3}-2 u P\right\} d \xi=0 .
$$

The last equality is justified because $\lim _{|\xi| \rightarrow \infty} u(\xi)=0$, while $P$ is uniformly bounded. This proves our claim, namely

$$
\begin{aligned}
\widetilde{E}(t) & =\int_{\mathbb{R}}\left(u^{2}(t, \xi) \cos ^{2} \frac{v(t, \xi)}{2}+\sin ^{2} \frac{v(t, \xi)}{2}\right) q(t, \xi) d \xi \\
& =\widetilde{E}(0) \doteq E_{0}
\end{aligned}
$$

along any solution of (2.18).

As long as the solution is defined, using (4.2) and (4.11) we obtain the bound

$$
\sup _{\xi \in \mathbb{R}}\left|u^{2}(t, \xi)\right| \leq 2 \int_{\mathbb{R}}\left|u u_{\xi}\right| d \xi \leq 2 \int_{\mathbb{R}}|u| \cdot\left|\sin \frac{v}{2} \cos \frac{v}{2}\right| q d \xi \leq E_{0} .
$$

This provides a uniform a priori bound on $\|u(t)\|_{L^{\infty}}$. Recalling the definitions (2.16)-(2.17), from the estimate (4.11) we recover the uniform bounds

$$
\|P(t)\|_{L^{\infty}} \leq C_{0}, \quad\left\|P_{x}(t)\right\|_{L^{\infty}} \leq C_{0}
$$

where the constant $C_{0}$ depends only on the total energy $E_{0}$.

Looking at the third equation in (2.18), by (4.12)-(4.13) we deduce that, as long as the solution is defined,

$$
\left|\frac{\partial q}{\partial t}\right| \leq\left(E_{0}+\frac{1}{2}+C_{0}\right) q
$$

Since $q(0, \xi) \equiv 1$, the previous differential inequality yields

$$
\exp \left\{-\left(E_{0}+\frac{1}{2}+C_{0}\right) t\right\} \leq q(t) \leq \exp \left\{\left(E_{0}+\frac{1}{2}+C_{0}\right) t\right\} .
$$

By the second equation in (2.18) it is clear that

$$
-\pi \leq v(t, \xi)<\pi .
$$

Finally, the lower bound on $q$ at (4.14) together with the energy estimate (4.11) imply that, for every $\eta>0$, there exists a constant $C_{\eta}$ depending only on $C_{0}, E_{0}$ and $T$ such that

$$
\operatorname{meas}(\{\xi ;|v(t, \xi)| \geq \eta\}) \leq C_{\eta} \quad t \in[0, T] .
$$

According to (4.12), (4.13) and (4.16), the a priori bounds (3.7)-(3.8) which we needed to construct a local solution are satisfied with a constant $C$ uniformly valid over any given time interval $[0, T]$. Therefore, the local solution can be globally extended for all times $t \geq 0$.

\section{5 - Continuous dependence}


The local existence theorem proved in Section 3 was obtained by representing the solution of (3.1)-(3.4) as the fixed point of a contraction in a suitable $\mathbf{L}^{\infty}$ space. This yielded uniqueness and continuous dependence w.r.t. to convergence on the initial data in $\mathbf{L}^{\infty}$. Our eventual goal is to show the continuous dependence of solutions to the Camassa-Holm equation (1.1), as the initial data converge in the $H^{1}$ norm. This requires further estimates. Indeed, if $\bar{u}_{n} \rightarrow \bar{u}$ in $H^{1}(\mathbb{R})$, setting $v=2 \arctan u_{x}$ one has

$$
\left\|u_{n}-\bar{u}\right\|_{\mathbf{L}^{\infty}} \rightarrow 0, \quad\left\|\bar{v}_{n}-\bar{v}\right\|_{\mathbf{L}^{2}} \rightarrow 0 .
$$

However, $\left\|\bar{v}_{n}-\bar{v}\right\|_{\mathbf{L}^{\infty}}$ does not converge to zero, in general. In this section we prove a result on continuous dependence of solutions of (2.18), based on the weaker assumptions (5.1).

Theorem 2. Consider a sequence of initial data $\bar{u}_{n}$ converging to $\bar{u}$ in $H^{1}(\mathbb{R})$. Let $u_{n}, u$ be the corresponding solutions of (2.18) with initial data (2.14). Then, for any $T>0$, the convergence

$$
u_{n}(t, \xi) \rightarrow u(t, \xi)
$$

holds uniformly for $(t, \xi) \in[0, T] \times \mathbb{R}$.

Proof. 1. Let $(u, v, q)$ and $(\tilde{u}, \tilde{v}, \tilde{q})$ be any two solutions of (2.18), corresponding to initial data of the form (2.14). Call $E_{0}$ an upper bound for the energies of the two solutions. Assuming that at time $t=0$

$$
\|u(0)-\tilde{u}(0)\|_{\mathbf{L}^{\infty}} \leq \delta_{0}, \quad\|v(0, \xi)-\tilde{v}(0, \xi)\|_{\mathbf{L}^{2}} \leq \delta_{0},
$$

we shall establish an a-priori bound on

$$
\|u(t)-\tilde{u}(t)\|_{\mathbf{L}^{\infty}} \quad \text { for } \quad t \in[0, T],
$$

depending only on $\delta_{0}, T$ and $E_{0}$.

2. Define the set

$$
\Lambda \doteq\{\xi \in \mathbb{R} ; \quad v(T, \xi)=-\pi\} \cup\{\xi \in \mathbb{R} ; \quad \tilde{v}(T, \xi)=-\pi\}
$$

Notice that

$$
\alpha^{*} \doteq \operatorname{meas}(\Lambda)
$$

is a number uniformly bounded by a constant depending only on $T$ and $E_{0}$.

3. For each $\xi \in \Lambda$, let $\tau(\xi)$ be the first time at which one of the two solutions reaches the value $-\pi$, namely

$$
\tau(\xi) \doteq \inf \{t \in[0, T] ; \quad \min \{v(t, \xi), \tilde{v}(t, \xi)\}=-\pi\} .
$$

Since the map $\xi \mapsto \tau(\xi)$ is measurable, we can construct a measure-preserving, measurable map $\alpha \mapsto \xi(\alpha)$ from the interval $\left[0, \alpha^{*}\right]$ onto the set $\Lambda$, having the additional property

$$
\alpha \leq \alpha^{\prime} \quad \text { if and only if } \quad \tau(\xi(\alpha)) \geq \tau\left(\xi\left(\alpha^{\prime}\right)\right) \text {. }
$$

The inverse mapping: $\Lambda \mapsto\left[0, \alpha^{*}\right]$, which is of course still measure-preserving, will be denoted as $\xi \mapsto \alpha(\xi)$. 
4. In connection with the map $\left[0, \alpha^{*}\right] \mapsto \Lambda$ considered at (5.7), we now define the distance functional

$$
\begin{aligned}
J((u, v, q),(\tilde{u}, \tilde{v}, \tilde{q})) \doteq\left(\|u-\tilde{u}\|_{\mathbf{L}^{\infty}}+\|v-\tilde{v}\|_{\mathbf{L}^{2}}+\|q-\tilde{q}\|_{\mathbf{L}^{2}}\right) & \\
& \quad+K_{0} \int_{0}^{\alpha^{*}} e^{K \alpha}|v(\xi(\alpha))-\tilde{v}(\xi(\alpha))| d \alpha \\
\doteq & J^{*}+K_{0} J^{\#} .
\end{aligned}
$$

For notational convenience, set

$$
J(t) \doteq J((u(t), v(t), q(t)),(\tilde{u}(t), \tilde{v}(t), \tilde{q}(t)))=J^{*}(t)+K_{0} J^{\#}(t) .
$$

We claim that, for some constants $K_{0}, K, M$ large enough (depending only on $T$ and $E_{0}$ ), one has

$$
\frac{d}{d t} J(t) \leq M \cdot J(t)
$$

This will of course imply

$$
J(t) \leq e^{M t} J(0) \quad t \in[0, T]
$$

thus providing an a-priori estimate on the distance at (5.4).

5. To complete the proof, we need to show that (5.10) holds, for suitable constants $K_{0}, K, M$ depending only on $T$ and $E_{0}$.

For each fixed $t \in[0, T]$, define the sets

$$
\begin{aligned}
\Gamma(t) & \doteq\{\xi \in \Lambda: \quad v(t, \xi)>-\pi, \quad \tilde{v}(t, \xi)=-\pi\} \cup\{\xi \in \Lambda: \quad v(t, \xi)=-\pi, \quad \tilde{v}(t, \xi)>-\pi\}, \\
\Gamma^{+}(t) & \doteq\{\xi \in \Lambda: \quad v(t, \xi)=\tilde{v}(t, \xi)=-\pi\}, \\
\Gamma^{-}(t) & \doteq\{\xi \in \Lambda: \quad v(t, \xi)>-\pi, \quad \tilde{v}(t, \xi)>-\pi\}=\{\xi \in \Lambda: \quad \tau(\xi)>t\} .
\end{aligned}
$$

Notice that the above three sets are disjoint, and

$$
\Gamma(t) \cup \Gamma^{+}(t) \cup \Gamma^{-}(t)=\Lambda \quad \text { for each } t \in[0, T] .
$$

Moreover, setting

$$
m(t) \doteq \operatorname{meas}\left(\Gamma^{-}(t)\right)
$$

the property (5.7) implies

$$
\Gamma^{-}(t)=\{\xi(\alpha) ; \quad \alpha \in[0, m(t)]\}
$$

6. As in the proof of Theorem 1, from the equations (2.18) we derive the estimate

$$
\begin{aligned}
& \frac{d}{d t}\left(\|u(t)-\tilde{u}(t)\|_{\mathbf{L}^{\infty}}+\|v(t)-\tilde{v}(t)\|_{\mathbf{L}^{2}}+\|q(t)-\tilde{q}(t)\|_{\mathbf{L}^{2}}\right) \\
& \quad \leq \kappa \cdot\left(\|u(t)-\tilde{u}(t)\|_{\mathbf{L}^{\infty}}+\|v(t)-\tilde{v}(t)\|_{\mathbf{L}^{2}}+\|q(t)-\tilde{q}(t)\|_{\mathbf{L}^{2}}+\operatorname{meas}(\Gamma(t))\right)
\end{aligned}
$$


7. Next, using (5.14) we compute

$$
\begin{gathered}
\frac{d}{d t} \int_{0}^{\alpha^{*}} e^{K \alpha}|v(t, \xi(\alpha))-\tilde{v}(t, \xi(\alpha))| d \alpha=\int_{\Gamma(t) \cup \Gamma^{-}(t) \cup \Gamma^{+}(t)} e^{K \alpha(\xi)} \cdot \frac{\partial}{\partial t}|v(t, \xi)-\tilde{v}(t, \xi)| d \xi \\
\quad=\int_{\Gamma(t)} e^{K \alpha(\xi)} \cdot \frac{\partial}{\partial t}|v(t, \xi)-\tilde{v}(t, \xi)| d \xi+\int_{0}^{m(t)} e^{K \alpha} \cdot \frac{\partial}{\partial t}|v(t, \xi(\alpha))-\tilde{v}(t, \xi(\alpha))| d \alpha .
\end{gathered}
$$

Indeed, the integral over $\Gamma^{+}(t)$ is zero.

As in (3.14), we can now choose $\delta>0$ small enough (depending only on $T, E_{0}$ ) such that, for $\xi \in \Gamma(t)$,

$$
|v(t, \xi)-\tilde{v}(t, \xi)| \leq \delta \quad \text { implies } \quad \frac{\partial}{\partial t}|v(t, \xi)-\tilde{v}(t, \xi)| \leq-\frac{1}{2} .
$$

On the other hand, choosing a constant $\kappa$ sufficiently large we easily obtain

$$
|v(t, \xi)-\tilde{v}(t, \xi)| \geq \delta \quad \text { implies } \quad \frac{\partial}{\partial t}|v(t, \xi)-\tilde{v}(t, \xi)| \leq-\frac{1}{2}+\kappa \cdot|v(t, \xi)-\tilde{v}(t, \xi)| .
$$

Finally, for $\xi \in \Gamma^{-}(t)$, we have the estimate

$$
\begin{aligned}
\frac{\partial}{\partial t}|v(t, \xi)-\tilde{v}(t, \xi)| \leq \kappa \cdot\left(\|u(t)-\tilde{u}(t)\|_{\mathbf{L}^{\infty}}\right. & +\|v(t)-\tilde{v}(t)\|_{\mathbf{L}^{2}}+\|q(t)-\tilde{q}(t)\|_{\mathbf{L}^{2}} \\
& +\operatorname{meas}(\Gamma(t))+|v(t, \xi)-\tilde{v}(t, \xi)|)
\end{aligned}
$$

so that

$$
\begin{aligned}
& \int_{0}^{m(t)} e^{K \alpha} \cdot \frac{\partial}{\partial t}|v(t, \xi(\alpha))-\tilde{v}(t, \xi(\alpha))| d \alpha \leq \kappa\left(J^{*}(t)+\operatorname{meas}(\Gamma(t))\right) \int_{0}^{m(t)} e^{K \alpha} d \alpha \\
&+\kappa \int_{0}^{m(t)} e^{K \alpha}|v(t, \xi(\alpha))-\tilde{v}(t, \xi(\alpha))| d \alpha \\
& \leq \kappa\left(J^{*}(t)+\operatorname{meas}(\Gamma(t))\right) \int_{0}^{m(t)} e^{K \alpha} d \alpha+\kappa \int_{\Gamma^{-}(t)} e^{K \alpha(\xi)}|v(t, \xi)-\tilde{v}(t, \xi)| d \xi .
\end{aligned}
$$

8. We rewrite (5.15) in the form

$$
\frac{d}{d t} J^{*}(t) \leq \kappa \cdot\left(J^{*}(t)+\operatorname{meas}(\Gamma(t))\right) .
$$

Moreover, observing that

$$
\xi \in \Gamma(t) \quad \text { implies } \quad \alpha(\xi) \geq m(t),
$$

from (5.16)-(5.19) we deduce

$$
\begin{gathered}
\frac{d}{d t} J^{\#}(t) \leq-\frac{1}{2} \int_{\Gamma(t)} e^{K \alpha(\xi)} d \xi+\kappa \int_{\Gamma(t) \cup \Gamma^{-}(t)} e^{K \alpha(\xi)}|v(t, \xi)-\tilde{v}(t, \xi)| d \xi \\
+\kappa \cdot\left(J^{*}(t)+\operatorname{meas}(\Gamma(t))\right) \cdot \int_{0}^{m(t)} e^{K \alpha} d \alpha \\
\leq-\frac{1}{2} e^{K m(t)} \cdot \operatorname{meas}(\Gamma(t))+\kappa J^{\#}(t)+\kappa J^{*}(t) \int_{0}^{\alpha^{*}} e^{K \alpha} d \alpha \\
+\kappa \cdot \operatorname{meas}(\Gamma(t)) e^{K m(t)} \int_{0}^{m(t)} e^{K(\alpha-m(t))} d \alpha \\
\leq-\frac{1}{4} e^{K m(t)} \cdot \operatorname{meas}(\Gamma(t))+\kappa J^{\#}(t)+\frac{\kappa}{K} e^{K \alpha^{*}} J^{*}(t)
\end{gathered}
$$


provided that we choose the constant $K \doteq 4 \kappa$, so that

$$
\kappa \int_{0}^{m(t)} e^{K(\alpha-m(t))} d \alpha \leq \frac{\kappa}{K}=\frac{1}{4}
$$

9. In the definition of the distance functional at (5.8)-(5.9) we now choose $K_{0} \doteq 4 \kappa$. Combining (5.20) and (5.21) we obtain

$$
\begin{aligned}
\frac{d}{d t}\left(J^{*}(t)+4 \kappa J^{\#}(t)\right) & \leq \kappa \cdot\left(J^{*}(t)+\operatorname{meas}(\Gamma(t))\right)+4 \kappa \cdot\left(-\frac{1}{4} \operatorname{meas}(\Gamma(t))+\kappa J^{\#}(t)+\frac{\kappa}{K} e^{K \alpha^{*}} J^{*}(t)\right) \\
& \leq \kappa J^{*}(t)+4 \kappa^{2} J^{\#}(t)+\kappa e^{4 \kappa \alpha^{*}} J^{*}(t) .
\end{aligned}
$$

This proves the differential inequality (5.10), with $M=\kappa+\kappa e^{4 \kappa \alpha^{*}}$.

\section{6 - Global dissipative solutions to the Camassa-Holm equation}

Reverting to the original variables $(t, x)$, we now show that the global solution of the system (2.18) yields a global dissipative solution to the Camassa-Holm equation (1.1). Given any $\bar{u} \in$ $H^{1}(\mathbb{R})$, let $(u, v, q)$ be the corresponding solution of (2.18) with initial data (2.14). Define

$$
y(t, \xi) \doteq \bar{y}(\xi)+\int_{0}^{t} u(\tau, \xi) d \tau .
$$

For each fixed $\xi$, the function $t \mapsto y(t, \xi)$ thus provides a solution to the Cauchy problem

$$
\frac{\partial}{\partial t} y(t, \xi)=u(t, \xi), \quad y(0, \xi)=\bar{y}(\xi) .
$$

We claim that a solution of (1.1) can be obtained by setting

$$
u(t, x) \doteq u(t, \xi) \quad \text { if } y(t, \xi)=x .
$$

Theorem 3. Let $(u, v, q)$ provide a global solution to the Cauchy problem (2.18), (2.14). Then the function $u=u(t, x)$ defined by (6.1), (6.3) provides a solution to the initial value problem (1.1)-(1.2) for the Camassa-Holm equation.

Proof. The argument given here relies on similar techniques as in [BC2].

1. By (4.12) we have the uniform bound $|u(t, \xi)| \leq E_{0}^{1 / 2}$. Hence (6.1) implies

$$
\bar{y}(\xi)-E_{0}^{1 / 2} t \leq y(t, \xi) \leq \bar{y}(\xi)+E_{0}^{1 / 2} t
$$

For each $t$, this yields

$$
\lim _{\xi \rightarrow \pm \infty} \bar{y}(t, \xi)= \pm \infty .
$$

Therefore, the image of the continuous map $(t, \xi) \mapsto(t, y(t, \xi))$ covers the entire domain $[0, \infty[\times \mathbb{R}$. 
2. For reader's convenience, we collect here the basic relations between the $(t, x)$ and the $(t, \xi)$ variables.

$$
\begin{array}{crr}
\frac{\partial u}{\partial \xi}(t, \xi)=\frac{q(t, \xi)}{2} \sin v(t, \xi), & \frac{\partial y}{\partial \xi}(t, \xi)=q(t, \xi) \cos ^{2} \frac{v(t, \xi)}{2}, \\
u_{x}(t, x)=\frac{\sin v(t, \xi)}{1+\cos v(t, \xi)} \quad \text { if } \quad x=y(t, \xi), \cos v(t, \xi) \neq-1 .
\end{array}
$$

Notice that (6.4) is consistent with the fact that $u_{\xi}=y_{\xi}=0$ when $v=-\pi$.

By the second equality in (6.4), for each fixed $t \geq 0$ the map $\xi \mapsto y(t, \xi)$ is non-decreasing. Moreover, if $\xi<\xi^{\prime}$ but $y(t, \xi)=y\left(t, \xi^{\prime}\right)$, then

$$
\int_{\xi}^{\xi^{\prime}} y_{\xi}(t, s) d s=\int_{\xi}^{\xi^{\prime}} q(t, s) \cos ^{2} \frac{v(t, s)}{2} d s=0 .
$$

Hence $\cos (v / 2) \equiv 0$ throughout the interval of integration. The first equality in (6.4) yields

$$
u\left(t, \xi^{\prime}\right)-u(t, \xi)=\int_{\xi}^{\xi^{\prime}} \frac{q(t, s)}{2} \sin v(t, s) d s=0 .
$$

Therefore the the map $(t, x) \mapsto u(t, x)$ at $(6.3)$ is well defined, for all $(t, x) \in[0, \infty[\times \mathbb{R}$.

3. We now observe that, for every fixed $t$, the image of the singular set where $v=-\pi$ has measure zero (in the $x$-variable). Indeed

$$
\begin{aligned}
\operatorname{meas}(\{y(t, \xi) ; v(t, \xi) & =-\pi\})=\int_{\{v(t, \xi)=-\pi\}} y_{\xi}(t, \xi) d \xi \\
& =\int_{\{v(t, \xi)=-\pi\}} q(t, \xi) \cos ^{2} \frac{v(t, \xi)}{2} d \xi=0
\end{aligned}
$$

Next, using (6.5) to change the variable of integration, we compute

$$
\begin{aligned}
& \int_{\mathbb{R}}\left(u^{2}(t, x)+u_{x}^{2}(t, x)\right) d x \\
& \quad=\int_{\{v(t, x)>-\pi\}}\left(u^{2}(t, \xi) \cos ^{2} \frac{v(t, \xi)}{2}+\sin ^{2} \frac{v(t, \xi)}{2}\right) q(t, \xi) d \xi \leq E_{0},
\end{aligned}
$$

because of (4.11). By a Sobolev inequality [EG], this implies the uniform Hölder continuity with exponent $1 / 2$ of $u$ as a function of $x$. By the first equation in (2.18) and the uniform bound on $\left\|P_{x}\right\|_{\mathbf{L} \infty}$, it follows that the map $t \mapsto u(t, y(t, \xi))$ is uniformly Lipschitz continuous along every characteristic curve $t \mapsto y(t, \xi)$. Therefore, $u=u(t, x)$ is globally Hölder continuous on the entire $t-x$ plane.

4. We now prove that the map $t \mapsto u(t)$ is Lipschitz continuous with values in $\mathbf{L}^{2}(\mathbb{R})$. Indeed, consider any interval $[\tau, \tau+h]$. For a given point $x$, choose $\xi \in \mathbb{R}$ such that the characteristic $t \mapsto y(t, \xi)$ passes through the point $(\tau, x)$. By (3.1) and the bound (3.27) it follows

$$
\begin{aligned}
|u(\tau+h, x)-u(\tau, x)| & \leq|u(\tau+h, x)-u(\tau+h, y(\tau+h, \xi))|+|u(\tau+h, y(\tau+h, \xi))-u(\tau, x)| \\
& \leq \sup _{|y-x| \leq E_{0}^{1 / 2} h}|u(t+h, y)-u(\tau+h, x)| d x+\int_{\tau}^{\tau+h}\left|P_{x}(t, \xi)\right| d t .
\end{aligned}
$$


Integrating over the whole real line we obtain

$$
\begin{aligned}
\int_{\mathbb{R}} & |u(\tau+h, x)-u(\tau, x)|^{2} d x \\
& \leq 2 \int_{\mathbb{R}}\left(\int_{x-E_{0}^{1 / 2} h}^{x+E_{0}^{1 / 2} h}\left|u_{x}(\tau+h, y)\right| d y\right)^{2} d x+2 \int_{\mathbb{R}}\left(\int_{\tau}^{\tau+h}\left|P_{x}(t, \xi)\right| d t\right)^{2} q(\tau, \xi) \cos ^{2} \frac{v(\tau, \xi)}{2} d \xi \\
& \leq 2 \int_{\mathbb{R}}\left(2 E_{0}^{1 / 2} h \int_{x-E_{0}^{1 / 2} h}^{x+E_{0}^{1 / 2} h}\left|u_{x}(\tau+h, y)\right|^{2} d y\right) d x+2 \int_{\mathbb{R}}\left(h \int_{\tau}^{\tau+h}\left|P_{x}(t, \xi)\right|^{2} d t\right)\|q(\tau)\|_{\mathbf{L}^{\infty} d \xi} \\
& =4 E_{0}^{1 / 2} h \int_{\mathbb{R}} \int_{y-E_{0}^{1 / 2} h}^{y+E_{0}^{1 / 2} h}\left|u_{x}(\tau+h, y)\right|^{2} d x d y+2 h\|q(\tau)\|_{\mathbf{L}^{\infty}} \int_{\mathbb{R}} \int_{\tau}^{\tau+h}\left|P_{x}(t, \xi)\right|^{2} d t d \xi \\
& \leq 8 E_{0} h^{2}\left\|u_{x}(\tau+h)\right\|_{\mathbf{L}^{2}}^{2}+2 h\|q(\tau)\|_{\mathbf{L}^{\infty}} \int_{\tau}^{\tau+h}\left\|P_{x}(t)\right\|_{\mathbf{L}^{2}}^{2} d t \\
& \leq C h^{2}
\end{aligned}
$$

for some constant $C$ uniformly valid as $t$ ranges on bounded set, in view of (3.14), (3.29) and (4.8). This clearly implies the Lipschitz continuity of the map $t \mapsto u(t)$, in terms of the $x$-variable.

5. Since $\mathbf{L}^{2}(\mathbb{R})$ is a reflexive space, in view of the infinite-dimensional version of Rademacher's theorem $[\mathrm{A}]$ the map $t \mapsto u(t)$ is differentiable for a.e. $t \in \mathbb{R}$. Since the right hand side of (1.3) clearly lies in $\mathbf{L}^{2}(\mathbb{R})$, to establish the equality it suffices to prove the following. For every smooth function with compact support $\phi \in \mathcal{C}_{c}^{\infty}$, at almost every time $t$ one has

$$
\begin{aligned}
\frac{d}{d t} \int u(t, x) \phi(x) d x & =\int\left(-u(t, x) u_{x}(t, x)-P_{x}(t, x)\right) \phi(x) d x \\
& =\int\left(u^{2}(t, x) \phi^{\prime}(x)-P_{x}(t, x) \phi(x)+u(t, x) u_{x}(t, x) \phi(x)\right) d x .
\end{aligned}
$$

Toward this goal, for each $\xi \in \mathbb{R}$ define

$$
\tau(\xi) \doteq \inf \{t>0 ; \quad v(t)=-\pi\} .
$$

Observe that, for almost every time $t \geq 0$ one has

$$
\operatorname{meas}(\{\xi ; \tau(\xi)=t\})=0 .
$$

Choose a time $t$ such that (6.10) holds. Integrating w.r.t. the variable $\xi$ and recalling (6.4) and (2.5) we obtain

$$
\begin{aligned}
& \frac{d}{d t} \int u(t, \xi) \phi(y(t, \xi))\left[q(t, \xi) \cos ^{2} \frac{v(t, \xi)}{2}\right] d \xi \\
& \quad=\int\left\{u_{t} \phi q \cos ^{2} \frac{v}{2}+u \phi^{\prime} y_{t} q \cos ^{2} \frac{v}{2}+u \phi q_{t} \cos ^{2} \frac{v}{2}-u \phi q v_{t} \sin v\right\} d \xi \\
& =\int_{\{v(t, \xi)>-\pi\}}\left\{-P_{x} \phi q \cos ^{2} \frac{v}{2}+u^{2} \phi^{\prime} q \cos ^{2} \frac{v}{2}+u \phi\left(u^{2}+\frac{1}{2}-P\right) \sin v q \cos ^{2} \frac{v}{2}\right. \\
& \left.-u \phi q\left(2\left(u^{2}-P\right) \cos ^{2} \frac{v}{2}-\sin ^{2} \frac{v}{2}\right) \frac{\sin v}{2}\right\} d \xi \\
& =\int_{\{v(t, \xi)>-\pi\}}\left\{-P_{x} \phi q \cos ^{2} \frac{v}{2}+u^{2} \phi^{\prime} q \cos ^{2} \frac{v}{2}+\frac{1}{2} u \phi \sin v q\right\} d \xi \\
& =\int_{\{v(t, \xi)>-\pi\}}\left(-P_{x} \phi+u^{2} \phi^{\prime} q+u u_{x} \phi\right) q \cos ^{2} \frac{v}{2} d \xi
\end{aligned}
$$


This establishes (6.8), thus proving that $u$ is a solution of the Camassa-Holm equation in the sense of Definition 1.

\section{7 - A semigroup of dissipative solutions}

Given an initial data $\bar{u} \in H^{1}(\mathbb{R})$, we denote by $u(t)=S_{t} \bar{u}$ the corresponding global solution of the Camassa-Holm equation constructed in Theorem 3. Aim of this final section is to prove:

Theorem 4. The map $S: H^{1} \times\left[0, \infty\left[\mapsto H^{1}\right.\right.$ is a semigroup. In addition, the following properties hold.

(i) The total energy is a non-increasing function of time, namely

$$
\|u(t)\|_{H^{1}(\mathbb{R})} \leq\left\|u\left(t^{\prime}\right)\right\|_{H^{1}(\mathbb{R})} \quad \text { whenever } 0 \leq t^{\prime} \leq t .
$$

(ii) For a constant $C$, depending only on the total energy $\|\bar{u}\|_{H^{1}}$, one has

$$
u_{x}(t, x) \leq C \cdot\left(1+t^{-1}\right) \quad t>0, \quad x \in \mathbb{R} .
$$

(iii) Given a sequence of initial data $\bar{u}_{n}$ such that $\left\|\bar{u}_{n}-\bar{u}\right\|_{H^{1}(\mathbb{R})} \rightarrow 0$, the corresponding solutions $u_{n}=u_{n}(t, x)$ converge to $u(t, x)$ uniformly for $t, x$ in bounded sets.

Proof. 1. To prove (7.1), for each $\xi \in \mathbb{R}$ define $\tau(\xi)$ as in (6.9). Recalling (6.7) we compute

$$
\begin{aligned}
\|u(t)\|_{H^{1}} & =\int_{\{v(t, \xi)>-\pi\}}\left(u^{2}(t, \xi) \cos ^{2} \frac{v(t, \xi)}{2}+\sin ^{2} \frac{v(t, \xi)}{2}\right) q(t, \xi) d \xi \\
& =E_{0}-\int_{\{\tau(\xi) \leq t\}}\left(u^{2}(t, \xi) \cos ^{2} \frac{v(t, \xi)}{2}+\sin ^{2} \frac{v(t, \xi)}{2}\right) q(t, \xi) d \xi \\
& =E_{0}-\int_{\left\{\tau(\xi) \leq t^{\prime}\right\} \cup\left\{t^{\prime}<\tau(\xi) \leq t\right\}} \sin ^{2} \frac{v(\tau(\xi), \xi)}{2} \cdot q(\tau(\xi), \xi) d \xi \\
& \leq E_{0}-\int_{\left\{\tau(\xi) \leq t^{\prime}\right\}} \sin ^{2} \frac{v(\tau(\xi), \xi)}{2} \cdot q(\tau(\xi), \xi) d \xi \\
& =\left\|u\left(t^{\prime}\right)\right\|_{H^{1}} .
\end{aligned}
$$

2. We now prove the Oleinik type estimate (7.2). As in the proof of Theorem 1, we can choose $\delta>0$ so that

$$
v \in\left[\pi-\delta, \pi\left[\quad \text { implies } \quad \frac{\partial}{\partial t} v(t, \xi) \leq-\frac{1}{2} .\right.\right.
$$

Therefore

$$
v(t, \xi)<\min \left\{\pi-\delta, \pi-\frac{t}{2}\right\}
$$

By (6.5), this yields (7.2), for a suitable constant $C$. 
3. Next, let $\bar{u}_{n}$ be a sequence of initial data converging to $\bar{u}$ in $H^{1}(\mathbb{R})$. By Theorem 2 this this implies the convergence $u_{n}(t, \xi) \rightarrow u(t, \xi)$ uniformly for $t, \xi$ in compact sets. Returning to the original $t-x$ coordinates, we obtain the convergence

$$
y_{n}(t, \xi) \rightarrow y(t, \xi), \quad u(t, x) \rightarrow u_{n}(t, x),
$$

uniformly on bounded sets, because all functions $u, u_{n}$ are uniformly Hölder continuous.

4. To complete the proof, it remains to establish the semigroup property. Fix $\bar{u} \in H^{1}$ and $\tau>0$. For all $t>0$ we need to show that

$$
S_{t}\left(S_{\tau} \bar{u}\right)=S_{\tau+t} \bar{u} .
$$

Let $(t, \xi) \mapsto(u, v, q)(t, \xi)$ be the corresponding solution of (2.18) with initial data (2.14). Call $\hat{u} \doteq S_{\tau} \bar{u}$. To construct the trajectory $t \mapsto S_{t} u^{*}$, we consider a new energy variable $\sigma$ and define the $\operatorname{map} \xi \mapsto \sigma(\xi)$ as a solution to the O.D.E.

$$
\frac{d}{d \xi} \sigma(\xi)=\left\{\begin{array}{lll}
q(\tau, \xi) & \text { if } & v(\tau, \xi)>-\pi \\
0 & \text { if } & v(\tau, \xi)=-\pi
\end{array}\right.
$$

with initial data

$$
\sigma\left(\xi_{0}\right)=0 \text {. }
$$

Here the value $\xi_{0}$ is chosen so that $y\left(\tau, \xi_{0}\right)=0$. We then define

$$
\left\{\begin{array}{l}
\hat{u}(t, \sigma)=u(\tau+t, \xi(\sigma)), \\
\hat{v}(t, \sigma)=v(\tau+t, \xi(\sigma)), \\
\hat{q}(t, \sigma)=\frac{q(\tau+t, \xi(\sigma))}{q(\tau, \xi(\sigma))},
\end{array}\right.
$$

where $\sigma \mapsto \xi(\sigma)$ provides an a.e. inverse to the map in (7.5)-(7.6), say

$$
\xi\left(\sigma^{*}\right) \doteq \sup \left\{s ; \sigma(s) \leq \sigma^{*}\right\} \text {. }
$$

5. For every $\sigma$, we claim that the previous definitions imply

$$
\int_{0}^{y(\tau, \xi)}\left(1+u_{x}^{2}(\tau, x)\right) d x=\sigma(\xi) .
$$

Indeed, by (7.6) this is true when $\xi=\xi_{0}, \sigma=0$. Moreover, recalling (6.4) and (2.5) one obtains

$$
\frac{\partial}{\partial \xi} y(\tau, \xi) \cdot\left(1+u_{x}^{2}(\tau, y(\tau, \sigma(\xi)))=q(\tau, \xi)=\frac{d}{d \xi} \sigma(\xi) .\right.
$$

By an integration, in view of (7.5) this establishes (7.8).

6. To establish the semigroup property, it now suffices to show that the functions (7.7) provide a solution to the system (2.18). Toward this goal, we write the identities

$$
q(\tau+t, \xi) d \xi=\frac{\hat{q}(t, \sigma(\xi))}{q(\tau, \sigma(\xi))} \cdot \frac{d \sigma(\xi)}{d \xi} \cdot d \sigma=\hat{q}(t, \sigma(\xi)) d \sigma .
$$

These imply that the corresponding integral source terms in (2.18) satisfy

$$
\widehat{P}(t, \sigma)=P(\tau+t, \xi(\sigma)), \quad \widehat{P}_{x}(t, \sigma)=P_{x}(\tau+t, \xi(\sigma)) .
$$

The conclusion now follows from (7.7) and (7.10), because the third equation in (2.18) is linear w.r.t. the variable $q$. 
We remark that the semigroup $S$ generated by the Camassa-Holm equation is NOT continuous as a map with values in $H^{1}$. In fact, even a single trajectory $t \mapsto u(t, \cdot)$ may fail to be continuous as a map from $\left[0, \infty\left[\right.\right.$ into $H^{1}$. This happens, for example, when a peakon and an antipeakon of the same strength collide and completely annihilate each other.

Example (Peakon-antipeakon interaction). The Camassa-Holm equation admits for every speed $c \in \mathbb{R}$ the solitary wave solution $[\mathrm{CH}]$

$$
u_{c}(x, t)=c e^{-|x-c t|},
$$

called peakon. Notice that the amplitude of a peakon equals to its speed. The peakon-antipeakon interaction is expressed by the following Ansatz [CH]:

$$
u(t, x)=p_{1}(t) e^{-\left|x-q_{1}(t)\right|}+p_{2}(t) e^{-\left|x-q_{2}(t)\right|}, \quad x \in \mathbb{R}, \quad t \geq 0,
$$

with $p_{1}, q_{1}, p_{2}, q_{2}$ Lipschitz continuous such that $p_{1}(0)>0, q_{1}(0)<0$, and

$$
p_{1}(t)=-p_{2}(t), \quad q_{1}(t)=-q_{2}(t) .
$$

Letting

$$
p(t)=2 p_{1}(t), \quad q(t)=2 q_{1}(t),
$$

we see that (7.11) is a solution in the sense of Definition 1 if the variables $q(t)$ and $p(t)$ satisfy the following Hamiltonian system of ordinary differential equations with discontinuous right-hand side

$$
\left\{\begin{array}{l}
p^{\prime}=\frac{p^{2}}{2} e^{q}, \\
q^{\prime}=p\left(1-e^{q}\right),
\end{array}\right.
$$

with Hamiltonian

$$
\mathcal{H}=p^{2}\left(1-e^{q}\right) .
$$

interpreted in the sense of Carathéodory (see [BC2]). For some maximal time $T>0$ (the breaking time) we will have $p(t)>0$ and $q(t)<0$ on $[0, T)$, while

$$
\lim _{t \uparrow T} p(t)=\infty \quad \text { and } \quad \lim _{t \uparrow T} q(t)=0 .
$$

More precisely, with

$$
H_{0}^{2}=p^{2}(0)\left(1-e^{q(0)}\right)
$$

we have (see $[\mathrm{BC} 2])$

$$
\left\{\begin{aligned}
T & =\frac{1}{H_{0}} \ln \frac{p(0)+H_{0}}{p(0)-H_{0}}, \\
p(t) & =H_{0} \frac{\left[p(0)+H_{0}\right]+\left[p(0)-H_{0}\right] e^{H_{0} t}}{\left[p(0)+H_{0}\right]-\left[p(0)-H_{0}\right] e^{H_{0} t}}, \quad t \in[0, T), \\
q(t) & =q(0)-2 \ln \frac{\left[p(0)+H_{0}\right] e^{-H_{0} t / 2}+\left[p(0)-H_{0}\right] e^{H_{0} t / 2}}{2 p(0)}, \quad t \in[0, T) .
\end{aligned}\right.
$$

Therefore (see $[\mathrm{BC} 2])$

$$
\lim _{t \uparrow T} u(t, x)=0 \quad \text { for every } \quad x \in \mathbb{R},
$$


while the total amount of energy concentrated in the interval between the two extremes (crest and trough) of the wave (7.11) equals

$$
\int_{q_{1}(t)}^{q_{2}(t)}\left(u^{2}(t, x)+u_{x}^{2}(t, x)\right) d x=\frac{p^{2}(t)}{2}\left(1-e^{2 q(t)}\right), \quad t \in[0, T)
$$

with

$$
\lim _{t \uparrow T} \int_{q_{1}(t)}^{q_{2}(t)}\left(u^{2}(t, x)+u_{x}^{2}(t, x)\right) d x=H_{0}^{2}
$$

But

$$
\|u(t, \cdot)\|_{H^{1}}^{2}=p^{2}(t)\left(1-e^{q(t)}\right)=H_{0}^{2}, \quad t \in[0, T),
$$

so that, as $t$ approaches the breaking time $T$, an increasingly large portion of the energy is concentrated within the interval $\left[q_{1}(t), q_{2}(t)\right]$. In the limit $t \uparrow T$ all the energy becomes concentrated at the single point $x=0$. In [BC2] the solution was continued past the breaking time as a conservative solution by setting,

$$
u(T+\varepsilon, x)=-u(T-\varepsilon, x), \quad x \in \mathbb{R},
$$

for $\varepsilon>0$. That is, in the conservative scenario the peakon and the antipeakon pass trough each other at breaking time and no energy is lost in the process. We now show that within the dissipative framework total annihilation occurs at breaking time, namely

$$
u(t, x)=0 \quad \text { for } \quad t>T, x \in \mathbb{R} .
$$

Indeed, using (7.11)-(7.13) in combination with (2.2), for $t \in[0, T)$ and $\xi \geq 0$, we have

$$
\left\{\begin{array}{l}
y(t, \xi)=\ln \left(e^{\xi}+\int_{0}^{t} p(s) \sinh \frac{q(s)}{2} d s\right) \quad \text { if } \quad \xi>-\frac{q(0)}{2}, \\
y(t, \xi)=\ln \frac{\left(e^{\xi}+1\right)+\left(e^{\xi}-1\right) e^{-2 \int_{0}^{t} p(s) e^{\frac{q(s)}{2}} d s}}{\left(e^{\xi}+1\right)-\left(e^{\xi}-1\right) e^{-2 \int_{0}^{t} p(s) e^{\frac{q(s)}{2}} d s}} \text { if } 0<\xi<-\frac{q(0)}{2},
\end{array}\right.
$$

while

$$
y(t, 0)=0 \quad \text { and } \quad y\left(t,-\frac{q(0)}{2}\right)=-\frac{q(t)}{2} \quad \text { for } \quad t \in[0, T[.
$$

Using (2.3), we infer that for $t \in[0, T)$,

$$
v(t, \xi)=-2 \arctan \frac{p(t) \sinh \frac{q(t)}{2}}{e^{\xi}+\int_{0}^{t} p(s) \sinh \frac{q(s)}{2} d s} \quad \text { if } \quad \xi \geq-\frac{q(0)}{2},
$$

while

$$
v(t, \xi)=-2 \arctan \left(p(t) e^{\frac{q(t)}{2}} \frac{\left(e^{\xi}+1\right)^{2}+\left(e^{\xi}-1\right)^{2} e^{-4 \int_{0}^{t} p(s) e^{\frac{q(s)}{2}} d s}}{\left(e^{\xi}+1\right)^{2}-\left(e^{\xi}-1\right)^{2} e^{-4 \int_{0}^{t} p(s) e^{\frac{q(s)}{2}} d s}}\right) \quad \text { if } \quad 0<\xi<-\frac{q(0)}{2} .
$$

Since (7.17) ensures

$$
\lim _{t \uparrow T} \int_{0}^{t} p(s) \sinh \frac{q(s)}{2} d s=1-e^{-\frac{q(0)}{2}}, \quad \lim _{t \uparrow T} p(t) e^{\frac{q(t)}{2}}=\infty, \quad \lim _{t \uparrow T} p(t) \sinh \frac{q(t)}{2}=0,
$$


from the previous relations and their analogues for $\xi<0$ we deduce that

$$
u(t, \xi), u_{x}(t, \xi), v(t, \xi) \rightarrow 0 \quad \text { as } \quad t \uparrow T \quad \text { if } \quad \xi \neq 0
$$

while

$$
u(t, 0)=0 \quad \text { on } \quad[0, T) \quad \text { and } \quad \lim _{t \uparrow T} v(t, 0)=-\pi .
$$

Therefore the solution $U=(u, v, q)$ of $(2.18)$ at $t=T$ equals $(0,0,1)$ and by Theorem 1 we infer that $U(t)=(0,0,1)$ for all $t \geq T$. That is, for the peakon-antipeakon interaction in the dissipative case we have $u(t, \cdot) \equiv 0$ for $t \geq T$.

Acknowledgements. The work of the first author was supported by the N.S.F., Grant DMS0505430 - Applied Mathematics: Hyperbolic systems of conservation laws. The second author gratefully acknowledges the support of the G. Gustafsson Foundation for Research in Natural Sciences and Medicine.

\section{References}

[A] N. Aronszajn, Differentiability of Lipschitzian mappings between Banach spaces, Studia Math. 57 (1976), 147-190.

[BSS1] R. Beals, D. Sattinger and J. Szmigielski, Multi-peakons and a theorem of Stieltjes, Inverse Problems 15 (1999), L1-L4.

[BSS2] R. Beals, D. Sattinger and J. Szmigielski, Multipeakons and the classical moment problem, Adv. Math. 154 (2000), 229-257.

[BSS3] R. Beals, D. Sattinger and J. Szmigielski, Peakon-antipeakon interaction, J. Nonlinear Math. Phys. 8 (2001), 23-27.

[B] A. Bressan, Unique solutions for a class of discontinuous differential equations, Proc. Amer. Math. Soc. 104 (1988), 772-778.

[BC1] A. Bressan and A. Constantin, Global solutions of the Hunter-Saxton equation, SIAM J. Math. Anal. 37 (2005), 996-1026.

[BC2] A. Bressan and A. Constantin, Global conservative solutions of the Camassa-Holm equation, Arch. Rational Mech. Anal., to appear.

[BF] A. Bressan and M. Fonte, An optimal transportation metric for solutions of the Camassa-Holm equation, Methods Appl. Anal., to appear.

[BS] A. Bressan and W. Shen, Unique solutions of directionally continuous O.D.E's in Banach spaces, Analysis and Applications, to appear.

[BZZ] A. Bressan, P. Zhang and Y. Zheng, On asymptotic variational wave equations, Arch. Rat. Mech. Anal. (2006), to appear.

[BZ] A. Bressan, and Y. Zheng, Conservative solutions to a nonlinear variational wave equation Comm. Math. Phys. (2006), to appear. 
[CH] R. Camassa and D. D. Holm, An integrable shallow water equation with peaked solitons, Phys. Rev. Lett. 71 (1993), 1661-1664.

[CHK] G. M. Coclite, H. Holden and K. H. Karlsen, Global weak solutions to a generalized hyperelasticrod wave equation, SIAM J. Math. Anal. 37 (2005), 1044-1069.

[C1] A. Constantin, Existence of permanent and breaking waves for a shallow water equation: a geometric approach, Ann. Inst. Fourier (Grenoble) 50 (2000), 321-362.

[C2] A. Constantin, On the scattering problem for the Camassa-Holm equation, Proc. Roy. Soc. London Ser. A 457 (2001), 953-970.

[CE1] A. Constantin and J. Escher, Global existence and blow-up for a shallow water equation, Ann. Scuola Norm. Sup. Pisa 26 (1998), 303-328.

[CE2] A. Constantin and J. Escher, Wave breaking for nonlinear nonlocal shallow water equations, Acta Mathematica 181 (1998), 229-243.

[CM1] A. Constantin and H. P. McKean, A shallow water equation on the circle, Comm. Pure Appl. Math. 52 (1999), 949-982.

[CM2] A. Constantin and L. Molinet, Global weak solutions for a shallow water equation, Comm. Math. Phys. 211 (2000), 45-61.

[CS] A. Constantin and W. Strauss, Stability of peakons, Comm. Pure Appl. Math. 53 (2000), 603-610.

[EG] L. C. Evans and R. F. Gariepy, Measure Theory and Fine Properties of Functions, Studies in Advanced Mathematics, CRC Press, Boca Raton, FL, 1992.

[FF] A. Fokas and B. Fuchssteiner, Symplectic structures, their Bäcklund transformation and hereditary symmetries, Physica D 4 (1981), 47-66.

[I] R. Ivanov, Extended Camassa-Holm hierarchy and conserved quantities, Z. Naturforsch. 61a (2006), 133-138.

[J] R. S. Johnson, Camassa-Holm, Korteweg-de Vries and related models for water waves, J. Fluid Mech. 455 (2002), 63-82.

[L] J. Lenells, Conservation laws of the Camassa-Holm equation, J. Phys. A 38 (2005), 869-880.

[M] H. P. McKean, Fredholm determinants and the Camassa-Holm hierarchy, Comm. Pure Appl. Math. 56 (2003), 638-680.

[N] I. Natanson, Theory of Functions of a Real Variable, F. Ungar Publ. Co., New York, 1961.

[W] E. Wahlen, On the peakon-antipeakon interaction, Dynamics Cont. Discr. Impuls. Systems, to appear.

[XZ1 Z. Xin and P. Zhang, On the weak solutions to a shallow water equation, Comm. Pure Appl. Math. 53 (2000), 1411-1433. 
[XZ2] Z. Xin and P. Zhang, On the uniqueness and large time behavior of the weak solutions to a shallow water equation, Comm. Partial Differential Equations 27 (2002), 1815-1844. 